\title{
AN ERROR ANALYSIS FOR THE MIDPOINT METHOD
}

\author{
IOANNIS K. ARGYROS
}

\begin{abstract}
We approximate zeros of nonlinear operator equations in Banach space setting using Newton-Kantrorvich assumptions and the majorant theory for the midpoint method.
\end{abstract}

\section{Introduction}

Let $E_{1}, E_{2}$ be Banach spaces, and let $U\left(x_{0}, R\right)$ denote the closed ball with center $x_{0} \in E_{1}$ and of radius $R>0$ in $E_{1}$. Suppose that the nonlinear operator $F$ defined on an open convex subset $D$ of $E_{1}$ containing $U\left(x_{0}, R\right)$, with values in $E_{2}$, is Frechet differentiable at every interior point of $U\left(x_{0}, R\right)$ and satisfies the condition.

$$
\left\|F^{\prime}(x+h)-F^{\prime}(x)\right\| \leq A(r,\|h\|), \quad x \in U\left(x_{0}, r\right), \quad 0 \leq r \leq R, \quad 0 \leq\|h\| \leq R-r .
$$

Here $A$ is a nonnegative and continuous function of two variable such that if one of the variable is fixed then $A$ is a nondecreasing function of the other on a corresponding sub-interval of $[0, R]$.

Moreover, we assume that $\frac{\partial A(0, t)}{\partial t}$ is positive, continuous and nondecreasing on $[0, R-$ $r]$ with $A(0,0)=0$

Note that by setting $A(r,\|h\|)=c\|h\|$ for all $r,\|h\|$, for some $c \geq 0$, we obtain the usual Lipschitz conditions on $F^{\prime}$ (see, [1], [3]), whereas for $A(r,\|h\|)=e(r)\|h\|$, we obtain some generalized condiitons considered also in [4], [9], [16], [22] but for Newton methods. Conditions of the form (1) wher also considered in (3) for Newton's method.

Let $x_{0} \in D$ be arbitrary and define the midpoint method for all $n \geq 0$ by

$$
y_{n}=x_{n}-F^{\prime}\left(x_{n}\right)^{-1} F\left(x_{n}\right)
$$

and

$$
x_{n+1}=x_{n}-F^{\prime}\left(\frac{1}{2}\left(x_{n}+y_{n}\right)\right)^{-1} F\left(x_{n}\right) .
$$

For a background on the midpoint method one can refer to [7], and the references there.

Received May 30, 1996; revised February 24, 1999.

1991 Mathematics Subject Classification. 47H17, 65J15, 49M15.

Key words and phrases. Midpoint method, Banach space. 
Using the majorant theory, we will show that under certain Newton-Kantorovich assumptions on the pair $\left(F, x_{0}\right)$, the midpoint method converges to a locally unique zero $x^{*}$ of equation

$$
F(x)=0 .
$$

We also provide upper bounds on the distances $\left\|x_{n}-x^{*}\right\|$ and $\left\|y_{n}-x^{*}\right\|$ for all $n \geq 0$.

Finally, we show that our results improve earlier ones [11]-[22].

\section{Convergence Analysis}

It is convenient to introduce constants

$$
\begin{gathered}
\eta \geq\left\|y_{0}-x_{0}\right\|, \quad \beta \geq\left\|F^{\prime}\left(x_{0}\right)^{-1}\right\|, \quad t_{0}=0, \quad s_{0} \geq \eta, \quad t_{1} \geq s_{0}^{*}, \\
s_{0}^{*}=s_{0}+\frac{\beta A\left(t_{0}, \frac{1}{2}\left(t_{0}+s_{0}\right)\right)\left(s_{0}-t_{0}\right)}{1-\beta A\left(0, \frac{1}{2}\left(t_{0}+s_{0}\right)\right)}
\end{gathered}
$$

scalar iterations

$$
\begin{aligned}
& s_{n+1}=t_{n+1}+\frac{\beta}{1-\beta A\left(0, t_{n+1}\right)} P\left(t_{n}, s_{n}\right) \\
& t_{n+2}=s_{n+1}+\frac{\beta}{1-\beta A\left(0, \frac{1}{2}\left(t_{n+1}+s_{n+1}\right)\right)} A\left(t_{n+1}, \frac{1}{2}\left(t_{n+1}+s_{n+1}\right)\right)\left(s_{n+1}-t_{n+1}\right), \\
& P\left(t_{n}, s_{n}\right)=\frac{\beta}{1-\beta A\left(0, t_{n+1}\right)}\left[\int_{t_{n}}^{s_{n}} A\left(s_{n}, t\right) d t+A\left(t_{n}, \frac{1}{2}\left(s_{n}-t_{n}\right)\right)\left(s_{n}-t_{n}\right),\right. \\
& \left.+\int_{0}^{s_{n}-t_{n}} A\left(t_{n}, t\right) d t+\frac{\beta}{1-\beta A\left(0, \frac{1}{2}\left(t_{n}+s_{n}\right)\right)} A\left(s_{n}, \frac{1}{2}\left(s_{n}-t_{n}\right)\right) A\left(t_{n}, \frac{1}{2}\left(s_{n}-t_{n}\right)\right)\left(s_{n}-t_{n}\right)\right] \\
& \text { for all } n \geq 0
\end{aligned}
$$

and the function

$$
\begin{aligned}
T(r)= & t_{1}+\frac{\beta}{1-\beta A(0, r)}\left(\frac{\beta}{1-\beta A(0, r)} A(r, r)+1\right) \\
& \cdot\left[\int_{0}^{r} A(r, t) d t+A\left(r, \frac{r}{2}\right) r+\frac{\beta}{1-\beta A(0, r)} A^{2}\left(r, \frac{r}{2}\right) r\right] .
\end{aligned}
$$

We can now prove the main results.

Theorem 1. Let $F: D \subset E_{1} \rightarrow E_{2}$ be a nonlinear operator defined on some open convex subset $D$ of a Banach space $E_{1}$ with values in $E_{2}$. Assume.

(a) $F$ is Frechet-differentiable on $U\left(x_{0}, R\right) \subseteq D$ for some $x_{0} \in D, R>0$, and satisfies (1);

(b) the inverse of the linear operator $F^{\prime}\left(x_{0}\right)$ exists and $\left\|F^{\prime}\left(x_{0}\right)^{-1}\right\| \leq \beta, \beta>0$; 
(c) there exists a minimum nonnegative number $R_{1}$, with

$$
\begin{aligned}
& T\left(R_{1}\right) \leq R_{1} ; \\
& R_{1} \leq R
\end{aligned}
$$

(d) the following estimates are true:

$$
\begin{gathered}
\beta A\left(0, R_{1}\right)<1, \\
\frac{\beta}{R-R_{1}} \int_{R_{1}}^{R} A(0, t) d t<1 \text { if } R \neq R_{1} \text { or } \beta A\left(0, R_{1}\right)<1 \text { if } R=R_{1},
\end{gathered}
$$

and

(e) $U\left(x_{0}, R\right) \subseteq D$.

Then

(i) The sequence $\left\{t_{n}\right\}(n \geq 0)$ defined by (6)-(7) is monotonically increasing and bounded above by its limit $R_{1}$ for all $n \geq 0$;

(ii) Then midpoint method generated by (2)-(3) is well defined, remains in $U\left(x_{0}, R_{1}\right)$ for all $n \geq 0$ and converges to a unique zero $x^{*}$ of equation $F(x)=0$ in $U\left(x_{0}, R\right)$.

Moreover the following estimates are true:

$$
\left\|x_{n}-x^{*}\right\| \leq R_{1}-t_{n}
$$

and

$$
\left\|y_{n}-x^{*}\right\| \leq R_{1}-s_{n} \text { for all } n \geq 0
$$

Proof. (i) We will show that sequence $\left\{t_{n}\right\}(n \geq 0)$ is monotonically increasing and bounded above by $R_{1}$ and as such it converges to some $R_{2}$ with $R_{2} \leq R_{1}$ (by (10)). From (5)-(8) and (10) $t_{0} \leq s_{0} \leq t_{1} \leq s_{1} \leq t_{2}$. By assuming $t_{k} \leq s_{k} \leq t_{k+1}, k=0,1,2, \ldots, n$ we obtain $t_{k+1} \leq s_{k+1} \leq t_{k+2}$ from (6)-(8) and (12). Hence, $\left\{t_{n}\right\}(n \geq 0)$ is monotonically increasing. From (5) and (11) $t_{0} \leq t_{1} \leq R$, and from (7) for $n=0, t_{2} \leq T\left(R_{1}\right) \leq R_{1}$. Let us assume that $t_{k} \leq R_{1}$ for $k=0,1,2, \ldots, n+1$. Then from (6)-(8)

$$
\begin{aligned}
t_{n+2}= & t_{n+1}+\left[\frac{\beta}{1-\beta A\left(0, t_{n+1}\right)}+\frac{\beta}{1-\beta A\left[0, \frac{1}{2}\left(t_{n+1}+s_{n+1}\right)\right]} \frac{\beta}{1-\beta A\left(0, t_{n+1}\right)}\right. \\
& \left.\cdot A\left[t_{n+1}, \frac{1}{2}\left(t_{n+1}+s_{n+1}\right)\right]\right] P\left(t_{n}, s_{n}\right) \\
\leq & t_{n+1}+\frac{\beta}{1-\beta A\left(0, R_{1}\right)}\left[\frac{\beta}{1-\beta A\left(0, R_{1}\right)} A\left(R_{1}, R_{1}\right)+1\right] P\left(t_{n}, s_{n}\right) \\
\leq & \cdots \leq t_{1}+\frac{\beta}{1-\beta A\left(0, R_{1}\right)}\left(\frac{\beta}{1-\beta A\left(0, R_{1}\right)} A\left(R_{1}, R_{1}\right)+1\right) \\
& {\left[\sum_{j=0}^{n+1} \int_{s_{j}}^{t_{j}+1} A\left(R_{1}, t\right) d t+\sum_{j=0}^{n} A\left(R_{1}, R_{1}\right)\left(s_{j}-t_{j}\right)\right.}
\end{aligned}
$$




$$
\begin{aligned}
& \left.\sum_{j=0}^{n} \int_{0}^{s_{j}-t_{j}} A\left(R_{1}, t\right) d t+\frac{\beta}{1-\beta A\left(0, R_{1}\right)} A\left(R_{1}, R_{1}\right)^{2} \sum_{j=0}^{n}\left(s_{j}-t_{j}\right)\right] \leq T\left(R_{1}\right) \leq R_{1} \\
& (\operatorname{by}(10)) .
\end{aligned}
$$

Hence, $\left\{t_{n}\right\}(n \geq 0)$ is bounded above by $R_{1}$. Moreover we deduce $t_{k} \leq s_{k} \leq t_{k+1} \leq R_{1}$ for all $k \geq 0$.

That completes the proof of part (i).

(ii) We will show that if

$$
\begin{aligned}
& \left\|y_{n}-x_{n}\right\| \leq s_{n}-t_{n}, \quad n \geq 0 \\
& \left\|F\left(x_{n}\right)\right\| \leq P\left(t_{n-1}, s_{n-1}\right), \quad n \geq 1 \\
& \left\|F^{\prime}\left(x_{n+1}\right)^{-1}\right\| \leq \frac{\beta}{1-\beta A\left(0, t_{n+1}\right)}, \quad n \geq 0 \\
& \left\|F^{\prime}\left(\frac{1}{2}\left(x_{n}+y_{n}\right)\right)^{-1}\right\| \leq \frac{\beta}{1-\beta A\left(0, \frac{1}{2}\left(t_{n}+s_{n}\right)\right)}, \quad n \geq 0
\end{aligned}
$$

then

$$
\begin{aligned}
& \left\|x_{n+1}-y_{n}\right\| \leq t_{n+1}-s_{n} \\
& \left\|F\left(x_{n+1}\right)\right\| \leq P\left(t_{n}, s_{n}\right)
\end{aligned}
$$

and

$$
\left\|y_{n+1}-x_{n+1}\right\| \leq s_{n+1}-t_{n+1} \text { for all } n \geq 0 .
$$

From (3), (17) and (19) we obtain for $k=0, \ldots, n-1$,

$$
\begin{aligned}
\| x_{k+2} & -y_{k+1} \| \\
& \leq\left\|F^{\prime}\left(\frac{1}{2}\left(x_{k+1}+y_{k+1}\right)\right)^{-1}\right\|\left\|F^{\prime}\left(\frac{1}{2}\left(x_{k+1}+y_{k+1}\right)\right)-F^{\prime}\left(x_{k+1}\right)\right\|\left\|y_{k+1}-x_{k+1}\right\| \\
& \leq \frac{\beta}{1-\beta A\left(0, \frac{1}{2}\left(t_{k+1}+s_{k+1}\right)\right.} A\left(t_{k+1}, \frac{1}{2}\left(t_{k+1}+s_{k+1}\right)\right)\left(s_{k+1}-t_{k+1}\right)=t_{k+2}-s_{k+1} .
\end{aligned}
$$

Also, by (5) we get

$$
\begin{aligned}
\left\|x_{1}-x_{0}\right\| & \leq\left\|F^{\prime}\left(\frac{1}{2}\left(x_{0}+y_{0}\right)\right)^{-1}\right\| \cdot\left\|F^{\prime}\left(\frac{1}{2}\left(x_{0}+y_{0}\right)\right)-F^{\prime}\left(x_{0}\right)\right\|\left\|y_{0}-x_{0}\right\| \\
& \leq \frac{\beta A\left(t_{0}, \frac{1}{2}\left(t_{0}+s_{0}\right)\right)}{1-\beta A\left(0, \frac{1}{2}\left(t_{0}+s_{0}\right)\right)}\left(s_{0}-t_{0}\right) \leq t_{1}-s_{0} .
\end{aligned}
$$

Hence, (20) is true.

Using (1)-(3), (16)-(20) and the approximation

$$
F\left(x_{n+1}\right)=\int_{0}^{1}\left[F^{\prime}\left(y_{n}+t\left(x_{n+1}-y_{n}\right)\right)-F^{\prime}\left(y_{n}\right)\right]\left(x_{n+1}-y_{n}\right) d t
$$




$$
\begin{aligned}
& +\int_{0}^{1}\left[F^{\prime}\left(x_{n}+t\left(y_{n}-x_{n}\right)\right)-F^{\prime}\left(x_{n}\right)\right]\left(y_{n}-x_{n}\right) d t \\
- & {\left[F^{\prime}\left(\frac{1}{2}\left(x_{n}+y_{n}\right)\right)-F^{\prime}\left(x_{n}\right)\right]\left(y_{n}-x_{n}\right)-\left[F^{\prime}\left(y_{n}\right)-F^{\prime}\left(\frac{1}{2}\left(x_{n}+y_{n}\right)\right)\right] } \\
& F^{\prime}\left(\frac{1}{2}\left(x_{n}+y_{n}\right)\right)^{-1} \\
& \cdot\left[F^{\prime}\left(\frac{1}{2}\left(x_{n}+y_{n}\right)\right)-F^{\prime}\left(x_{n}\right)\right]\left(y_{n}-x_{n}\right),
\end{aligned}
$$

we can obtain by taking norms

$$
\begin{aligned}
& \left\|F\left(x_{n+1}\right)\right\| \\
\leq & \int_{0}^{1}\left\|F^{\prime}\left(y_{n}+t\left(x_{n+1}-y_{n}\right)\right)-F^{\prime}\left(y_{n}\right)\right\|\left\|x_{n+1}-y_{n}\right\| d t \\
& +\int_{0}^{1}\left\|F^{\prime}\left(x_{n}+t\left(y_{n}-x_{n}\right)\right)-F^{\prime}\left(x_{n}\right)\right\| \cdot\left\|y_{n}-x_{n}\right\| d t \\
& +\| F^{\prime}\left(\frac{1}{2}\left(x_{n}+y_{n}\right)-F^{\prime}\left(x_{n}\right)\|\cdot\| y_{n}-x_{n} \|\right. \\
& +\left\|F^{\prime}\left(\frac{1}{2}\left(x_{n}+y_{n}\right)\right)^{-1}\right\| \cdot\left\|F^{\prime}\left(y_{n}\right)-F^{\prime}\left(\frac{1}{2}\left(x_{n}+y_{n}\right)\right)\right\| \\
& \cdot\left\|F^{\prime}\left(\frac{1}{2}\left(x_{n}+y_{n}\right)\right)-F^{\prime}\left(x_{n}\right)\right\| \cdot\left\|y_{n}-x_{n}\right\| \\
\leq & \int_{0}^{1} A\left(s_{n},(1-t) s_{n}+t t_{n+1}\right) d t+\int_{0}^{1} A\left(t_{n}, t\left(s_{n}-t_{n}\right)\right)\left(s_{n}-t_{n}\right) d t \\
& +A\left(t_{n}, \frac{1}{2}\left(t_{n}+s_{n}\right)\right)\left(s_{n}-t_{n}\right) \\
& +\frac{\beta}{1-\beta A\left(0, \frac{1}{2}\left(t_{n}+s_{n}\right)\right)} A\left(s_{n}, \frac{1}{2}\left(t_{n}+s_{n}\right)\right) A\left(t_{n}, \frac{1}{2}\left(t_{n}+s_{n}\right)\right)\left(s_{n}-t_{n}\right)=P\left(t_{n}, s_{n}\right),
\end{aligned}
$$

where we have also used estimates

$$
\begin{aligned}
\| x_{n+1} & -x_{0}\|\leq\| x_{n+1}-y_{0}\|+\| y_{0}-x_{0}\|\leq\| x_{n+1}-y_{n}\|+\| y_{n}-y_{0}\|+\| y_{0}-x_{0} \| \\
\leq & \cdots \leq\left(t_{n+1}-s_{n}\right)+\left(s_{n}-s_{0}\right)+s_{0} \leq t_{n+1} \leq R_{1} \\
\| y_{n+1}- & x_{0}\|\leq\| y_{n+1}-y_{0}\|+\| y_{0}-x_{0}\|\leq\| y_{n+1}-x_{n+1} \| \\
& \quad+\left\|x_{n+1}-y_{n}\right\|+\left\|y_{n}-y_{0}\right\|+\left\|y_{0}-x_{0}\right\| \\
& \leq \cdots \leq\left(s_{n+1}-t_{n+1}\right)+\left(t_{n+1}-s_{n}\right)+\left(s_{n}-s_{0}\right) \leq s_{n+1} \leq R_{1}
\end{aligned}
$$

Hence (21) is true. From (2), (18) and (21)

$$
\begin{aligned}
\left\|y_{n+1}-x_{n+1}\right\| & \leq\left\|F^{\prime}\left(x_{n+1}\right)^{-1}\right\| \quad\left\|F\left(x_{n+1}\right)\right\| \\
& \leq \frac{\beta}{1-\beta A\left(0, t_{n+1}\right)} P\left(t_{n}, s_{n}\right)=s_{n+1}-t_{n+1},
\end{aligned}
$$

from which (22) follows. 
Moreover, from (1), (5), (12) and estimate

$$
\left\|F^{\prime}\left(x_{0}\right)^{-1}\right\| \cdot\left\|F^{\prime}\left(x_{n}\right)-F^{\prime}\left(x_{0}\right)\right\| \leq \beta A\left(0, t_{n}\right) \leq \beta A\left(0, R_{1}\right)<1
$$

it follows from the Banach lemma on invertible operators that $F^{\prime}\left(x_{n}\right)^{-1}$ exists and

$$
\left\|F^{\prime}\left(x_{n}\right)^{-1}\right\| \leq \frac{\left\|F^{\prime}\left(x_{0}\right)^{-1}\right\|}{1-\left\|F^{\prime}\left(x_{0}\right)^{-1}\right\| \cdot\left\|F^{\prime}\left(x_{n}\right)-F^{\prime}\left(x_{0}\right)\right\|} \leq \frac{\beta}{1-\beta A\left(0, t_{n}\right)} \text { for all } n \geq 0
$$

Furthermore, from the estimate

$$
\left\|x_{n}+y_{n}-2 x_{0}\right\| \leq\left\|x_{n}-x_{0}\right\|+\left\|y_{n}-x_{0}\right\| \leq t_{n}+s_{n},
$$

we obtain for all $n \geq 0$

$$
\left\|F^{\prime}\left(x_{0}\right)^{-1}\right\| \cdot\left\|F^{\prime}\left(\frac{1}{2}\left(x_{n}+y_{n}\right)\right)-F^{\prime}\left(x_{0}\right)\right\| \leq \beta A\left(0, \frac{1}{2}\left(t_{n}+s_{n}\right)\right) \leq \beta A\left(0, R_{1}\right)<1 .
$$

it now follows that $F^{\prime}\left(\frac{1}{2}\left(x_{n}+y_{n}\right)\right)^{-1}$ exists and

$$
\left\|F^{\prime}\left(\frac{1}{2}\left(x_{n}+y_{n}\right)\right)^{-1}\right\| \leq \frac{\beta}{1-\beta A\left(0, \frac{1}{2}\left(t_{n}+s_{n}\right)\right)}, \text { for all } n \geq 0 .
$$

Hence, iterates $\left\{x_{n}\right\}(n \geq 0)$ generated by (2)-(3) are well defined for all $n \geq 0$. Also, by (16) and (20)

$$
\left\|x_{n+1}-x_{n}\right\| \leq\left\|x_{n+1}-y_{n}\right\|+\left\|y_{n}-x_{n}\right\| \leq t_{n+1}-t_{n} \text { for all } n \geq 0
$$

it now follows from the above estimate and (i) that the sequence $\left\{x_{n}\right\}(n \geq 0)$ is Cauchy in a Banach space and as such it converges to some $x^{*} \in U\left(x_{0}, R_{1}\right)$, which by taking the limit as $n \rightarrow \infty$ in (2) becomes a zero of $F$ since $F\left(x^{*}\right)=0$. Moreover, by (23) and (24) $x_{n}, y_{n} \in U\left(x_{0}, R_{1}\right)$. The estimates (14) and (15) follow immediately from (i), (20) and (22).

Finally to show uniqueness, we assume there exists another zero $y^{*}$ of equation (4) in $U\left(x_{0}, R\right)$. Then from (1), (13) and (27), we obtain

$$
\begin{aligned}
& \left\|F^{\prime}\left(x_{0}\right)^{-1}\right\| \cdot \int_{0}^{1}\left\|F^{\prime}\left(y^{*}+t\left(x^{*}-y^{*}\right)\right)-F^{\prime}\left(x_{0}\right)\right\| d t \\
& \leq\left\|F^{\prime}\left(x_{0}\right)^{-1}\right\| \int_{0}^{1} A\left(0,(1-t)\left\|x_{0}-y^{*}\right\|+t\left\|x^{*}-x_{0}\right\|\right) d t<1, \quad \text { by (12) and (13). }
\end{aligned}
$$

It now follows from the above inequality that the linear operator $\int_{0}^{1} F^{\prime}\left(y^{*}+t\left(x^{*}-y^{*}\right)\right) d t$ is invertible. From this fact, and the approximation

$$
F\left(x^{*}\right)-F\left(y^{*}\right)=\int_{0}^{1} F^{\prime}\left(y^{*}+t\left(x^{*}-y^{*}\right)\right)\left(x^{*}-y^{*}\right) d t
$$


it follows that $x^{*}=y^{*}$.

That completes the proof of the theorem.

Remarks. (a) From the estimates

$$
\left\|x_{n}-y_{0}\right\| \leq\left\|x_{n}-y_{n}\right\|+\left\|y_{n}-y_{0}\right\| \leq\left(t_{n}-s_{n}\right)+\left(s_{n}-s_{0}\right) \leq t_{n}-\eta \leq R_{1}-\eta
$$

and

$$
\begin{aligned}
\left\|y_{n+1}-y_{0}\right\| & \leq\left\|y_{n+1}-x_{n+1}\right\|+\left\|x_{n+1}-y_{n}\right\|+\left\|y_{n}-y_{0}\right\| \\
& \leq\left(s_{n+1}-t_{n+1}\right)+\left(t_{n+1}-s_{n}\right)+\left(s_{n}-s_{0}\right) \\
& \leq s_{n+1}-\eta \leq R_{1}-\eta
\end{aligned}
$$

it follows that $x_{n}, y_{n} \in U\left(y_{0}, R_{1}-\eta\right)$ for all $n \geq 0$. Note also that $R_{1}$ is the unique positive zero of $T(r)-r=0$ in $\left(0, R_{1}\right]$ (by (10)).

(b) We can use the midpoint method to approximate nonlinear equations with nondifferentiable operators. Indeed, consider the equation

$$
F_{1}(x)=0
$$

where

$$
F_{1}(x)=F(x)+Q(x)
$$

with $F$ as before and $Q$ satisfying an estimate of the form

$$
\|Q(x+h)-Q(x)\| \leq B(r,\|h\|), \quad x \in U\left(x_{0}, R\right), \quad 0 \leq r \leq R, \quad 0 \leq\|h\| \leq R-r
$$

where $B$ is a nonnegative and continuous function of two variable such that if one of the variables is fixed then $B$ is a non-decreasing function of the other on a corresponding subinterval of $[0, R]$. Note that the differentiability of $Q$ is not assumed here. Moreover we assume $B$ is linear in the right hand side variable. Replace $F$ in (2) and (3) by $F_{1}$ and leave the Frechet-derivatives as they are. Define the sequences $\left\{\bar{t}_{n}\right\}$ and $\left\{\bar{s}_{n}\right\}(n \geq 0)$ as the corresponding $\left\{t_{n}\right\}$ and $\left\{s_{n}\right\}$ given (6) and (7) respectively. The change will be and extra term of the form $B\left(t_{n}, s_{n}-t_{n}\right)$ added in the definition of $P\left(t_{n}, s_{n}\right)$. Define $T_{1}$ by $T$ in (9) the insert inside the bracket the term $B(r, r)$. Then following the proof of the above theorem step by step we can show a similar theorem with identical hypotheses and conclusions, but holding for equation (30). (See, also [4], [9], [10], [22]).

(c) In [7] we showed the result (see also [9]).

Theorem 2. Let $F: D \subset E_{1} \rightarrow E_{2}, E_{1}, E_{2}$ be real Banach spaces, and $D$ be an open convex domain in $E_{1}$. Assume that $F$ has 2 2nd order continous Frechet derivatives on $D$ and that the following conditions are satisfied:

$$
\left\|F^{\prime}(x)-F^{\prime}(y)\right\| \leq I\|x-y\|,\left\|F^{\prime \prime}(x)\right\| \leq N\|x-y\|,
$$


for all $x, y \in D$

$$
\begin{aligned}
& \left\|F^{\prime}\left(x_{0}\right)^{-1}\right\| \leq \beta,\left\|y_{0}-x_{0}\right\| \leq \eta \\
& {\left[M^{2}+\frac{7 N}{6 \beta}\right]^{\frac{1}{2}} \leq K,} \\
& h=K \beta \eta \leq \frac{1}{2}
\end{aligned}
$$

and

$$
U\left(y_{0}, r_{1}-\eta\right) \subset D
$$

Moreover, we define

$$
\begin{aligned}
& g(t)=\frac{1}{2} K t^{2}-\frac{1}{\beta} t+\frac{\eta}{\beta}, \\
& r_{1}=\frac{1-\sqrt{1-2 h}}{h} \eta
\end{aligned}
$$

and

$$
\theta=\frac{1-\sqrt{1-2 h}}{1+\sqrt{1-2 h}}
$$

where $r_{1}$ is the smallest zero of the equation $g(t)=0$. Then the midpoint method $(2)-(3)$ is convergent. Also $x_{n}, y_{n} \in U\left(y_{0}, r_{1}-\eta\right)$, for all $n \in N_{0}$. The limit $x^{*}$ is the unique zero of the equation $F(x)=0$ in $U\left(x_{0}, r_{2}^{*}\right), r_{1} \leq r_{2}^{*}<r_{2}$ if $L=K($ or $M=K)$ and $r_{2}^{*}=r_{2}$ if $I<K($ or $M<K)$.

Moreover, we have the following error estimates and optimal error constants:

$$
\begin{aligned}
& \left\|x_{n}-x^{*}\right\| \leq r_{1}-t_{n}^{1}, \quad \text { for all } n \\
& \left\|y_{n}-x^{*}\right\| \leq r_{1}-s_{n}^{1}, \quad \text { for all } n
\end{aligned}
$$

and

$$
r_{1}-t_{n}=\frac{\left(1-\theta^{2}\right) \eta}{1-\theta^{3^{n}}} \theta^{3^{n}-1}
$$

where

$$
s_{n}^{1}=t_{n}^{1}-\frac{g\left(t_{n}^{1}\right)}{g^{\prime}\left(t_{n}^{1}\right)}, \quad t_{0}^{1}=0
$$

and

$$
t_{n+1}^{1}=s_{n}^{1}-\frac{g\left(t_{n}^{1}\right)}{g^{\prime}\left(\frac{1}{2}\left(t_{n}^{1}+s_{n}^{1}\right)\right)} \quad \text { for all } n \geq 0 .
$$

(d) Several sufficient conditions can be given to show for examples that under the hypotheses of theorems 1 and 2

$$
s_{n}-t_{n} \leq s_{n}^{1}-t_{n}^{1} \text { for all } n \geq 0 .
$$


On such condition can be

$\frac{\beta}{1-\beta A(0, r)}\left[\int_{0}^{r} A(r, t) d t+A\left(r, \frac{r}{2}\right) r+\frac{\beta}{1-\beta A(0, r)} A^{2}\left(r, \frac{r}{2}\right) r\right] \leq s_{1}^{1}-t_{1}^{1}$, or $\leq-\frac{g(r)}{g^{\prime}(r)}$ for all $r \in[0, R]$.

The details are left to the motivated reader (see, also [4], [9]).

(e) Note that the order of convergence for the midpoint is three, whereas for Newton's method only 2[1], [9], [13], [16].

(f) Similar theorems can be proved if $\|h\|$ in $C_{1}$ is replaced by a Holder continuouity condition of the form $\|h\|^{P}$ for some $p \in[0,1]$. (See, also [9]).

(g) The function $A$ can be chosen as

$$
A(r,\|h\|)=\sup _{\substack{x, y \in U\left(x_{0}, r\right) \\\|h\| \leq R-r}}\left\|F^{\prime}(x+h)-F^{\prime}(x)\right\|
$$

or

$$
A(r,\|h\|)=\int_{r}^{r+\|h\|} q(t) d t
$$

where $q(r)$ is a nondecreasing function on the interval $[0, R]$ satisfying

$$
\left\|F^{\prime}(x)-F^{\prime}(y)\right\| \leq q(r)\|x-y\|
$$

for all $x, y \in U\left(x_{0}, r\right)$ (see also [4], [9], [16], [22]).

One can refer to [5], [9] for some applications of these choices to the solution of nonlinear integral equations of Uryson-type.

(h) Finally, if the right hand side of condition (1) changes to $A(r, r+\|h\|)$, a new theorem similar to Theorem 1 can then esaily follow. Remarks similar to (a)-(g) for the new condition can we also follow.

\section{Applications}

(A) In this section we will first give an example for Theorem 2 (similarly we can work for Theorem 1). We first note that the midpoint is of order three. The most popular methods of order three are the method of tangent parabolas (or Euler-Chebysheff) and the method of tangent hyperbolas (or Chebysheff-Halley) [1], [5], [6], [9], [12], [14], [15], [17], [18], [20], [21]. In all the above references it is assumed that $N>0$, which means that these methods cannot apply to solve quadratic operator equations of the form

$$
P(x)=B(x, x)+L(x)+z,
$$

where $B, L$ are bounded quadratic and linear operators respectively with $z$ fixed in $E_{2}$. We then have that $P^{\prime}(x)=2 B(x)+L$ and $P^{\prime \prime}(x)=2 B$. Hence, we get $M=2\|B\|$ 
and $N=0$. Integral equations that can be formulated in the form $P(x)=0$ have bery important applications in radiative transfer [2], [3], [4], [10].

In this section we use the theorem to suggest new approaches to the solution of quadratic integral equations of the form

$$
x(s)=y(s)+\lambda x(s) \int_{0}^{1} q(s, t) x(t) d t
$$

in the space $E_{1}=C[0,1]$ of all functions continuous on the interval $[0,1]$, with norm

$$
\|x\|=\max _{0 \leq s \leq 1}|x(s)|
$$

Here we assume that $\lambda$ is a real number called the "albedo" for scattering and the kernel $q(s, t)$ is a continuous function of two variable $s, t$ with $0<s, t<1$ and satisfying

(i) $0<q(s, t)<1,0 \leq s, t \leq 1, q(0,0)=1$;

(ii) $q(s, t)+q(t, s)=1,0 \leq s, t \leq 1$.

The function $y(s)$ is a given continuous function defined on $[0,1]$, and finally $x(s)$ is the unknown function sought in $[0,1]$.

Equations of this type are closely related with the work of S. Chandrasekhar [10], (Novel prize of Rhysics, 1983), and arise in the theories of radiative transfer, neutron transport and in the kinetic theory of gasses, [2], [3], [9], [10].

There exists an extensive literature on equations like (32) under various assumptions on the kernel $q(s, t)$ and $\lambda$ is a real or complex number. One can refer to the recent work in [2], [3], [9], [10] and the references there. Here we demonstrate that the theorem via the iterative procedure (2)-(3) provides existence results for (32).

For simplicity (without loss of generality) we will assume that

$$
q(s, t)=\frac{s}{s+t} \quad \text { for all } \quad 0 \leq s, \quad t \leq 1, \quad q(0,0)=1 .
$$

Note that $q(s, t)$ so defined satisfies (i) and (ii) above.

Let us now choose $\lambda=.25, y(s)=1$ for all $s \in[0,1]$; and define the operator $P$ on $E_{1}$ by

$$
P(x)=\lambda x(s) \int_{0}^{1} \frac{s}{s+t} x(t) d t-x(s)+1 .
$$

Note that every zero of the equation $P(x)=0$ satisfies equation (32).

Set $x_{0}(s)=1$, use the definition of the first and second Frechet-derivatives of the operator $P$ to obtain using the theorem,

$$
\begin{aligned}
& K=M=2|\lambda| \max _{0 \leq s \leq 1}\left|\int_{0}^{1} \frac{s}{s+t} d t\right|=2|\lambda| \ln 2=.34657359, \\
& \beta=\left\|P^{\prime}(1)^{-1}\right\|=1.53039421
\end{aligned}
$$




$$
\begin{aligned}
\eta & \geq\left\|P^{\prime}(1)^{-1} P(1)\right\| \geq \beta \lambda \ln 2=.265197107 \\
h & =.140659011<.5 \\
r_{1} & =.28704852, \quad r_{2}=3.4837317
\end{aligned}
$$

and

$$
\theta=.08239685 \text {. }
$$

(For detailed computations, see also [2], [3], and [9].)

Therefore according to theorem 2 equation (32) has a solution $x^{*}$ and the two-point method (2)-(3) converges to $x^{*}$. Note that, the results obtained in [12], [14], [15], [17], [18], [20], [21] cannot apply here, since $N=0$.

(B) We will complete this study by providing an example that shows how to choose the constants $B, \eta$ and the functions $A$ and $T$, where $A$ is chosen as in (31).

Let us assume that $E_{1}=E_{2}=C=C[0,1]$ the space of continuous functions on $[0,1]$ equipped with the usual supremum norm. We consider Uryson-type nonlinear integral equations of the form

$$
F(x)(t)=x(t)-\int_{0}^{1} K(t, s, x(s)) d s .
$$

We assume for simplicity that $x_{0}=0$, and make use of the following standard result whose proof can be found for example in [5] or [9].

Theorem 3. The Lipschitz condition (31) for the Frechet-derivative $F^{\prime}$ of the operator (33) holds if and only if the second derivative $K_{u u}^{\prime \prime}(t, s, u)$ exist for all $t$ and almost all $s$ and $u$, and

$$
\sup _{t \in[0,1]} \int_{0}^{1} \sup _{|u| \leq r}\left|K_{u u}^{\prime \prime}(t, s, u)\right| d s<\infty
$$

Moreover, the left hand side in relation (34) is then the minimal Lipschitz constant $q(r)$ in $(31)$.

Moreover, the constants $\eta$ and $\beta$ are given by

$$
\eta=\sup _{t \in[0,1]}\left|\int_{0}^{1} K(t, s, 0) d s+\int_{0}^{1} r(t, s) \int_{0}^{1} K(s, p, 0) d p d s\right|
$$

and

$$
\beta=1+\sup _{t \in[0,1]} \int_{0}^{1}|r(t, s)| d s,
$$

where $r(s, t)$ is the resolvent kernel of equation

$$
h(t)=\int_{0}^{1} K_{u}^{\prime}(t, s, 0) h(s) d s=-\int_{0}^{1} K(t, s, 0) d s .
$$

Let us consider a simple example. Suppose that $K(t, s, u)=c_{1}(t) c_{2}(s) c_{3}(u)$ with two continuous functions $c_{1}$ and $c_{2}$, and $c_{3} \in C^{2}$. We set

$$
d_{1}=\int_{0}^{1} c_{2}(s) d s, \quad d_{2}=\int_{0}^{1} c_{1}(s) c_{2}(s) d s .
$$


Then relation (37) becomes

$$
h(t)=\left[c_{4}^{1} c_{3}^{1}(0)-d_{1} c_{3}(0)\right] c_{1}(t),
$$

where

$$
c_{4}^{\prime}=\int_{0}^{1} c_{2}(s) h(s) d s .
$$

Substituting relation (39) into (40), one may calculate $c_{4}^{\prime}$ and hence find the resolvent kernel $r(t, s)$ in case $d_{2} c_{3}^{\prime}(0)<1$, to get

$$
r(t, s)=\frac{c_{1}(t) c_{2}(t) c_{3}^{\prime}(0)}{1-d_{2} c_{3}^{\prime}(0)}
$$

Using relations (34)-(36), we obtain

and

$$
\begin{gathered}
q(t)=\left\|c_{1}\right\| d_{1} \sup _{\|u\| \leq r}\left|c_{3}^{\prime \prime}(u)\right|, \\
\eta=\frac{d_{1} c_{3}(0)}{1-d_{2} c_{3}^{\prime}(0)}\left\|c_{1}\right\|
\end{gathered}
$$

$$
\beta=1+\frac{d_{1} c_{3}(0)}{1-d_{2} c_{3}^{\prime}(0)}\left\|c_{1}\right\|
$$

Thus, in this case a complete and explicit computation of the function $T$ given by relation (9) is possible. As an example, let us choose

$$
c_{1}(t)=\frac{3}{10} t, \quad c_{2}(s)=\frac{2}{10} s \quad \text { and } \quad c_{3}(u)=\frac{1}{3} u^{3}+\frac{1}{10} u+1
$$

on $[0,1]$. Then using relations $(38),(41)-(44),(5)$ and (9) we get

$$
\begin{aligned}
& d_{1}=\frac{1}{10}, \quad d_{2}=\frac{2}{100}, \quad d_{2} c_{3}^{1}(0)=\frac{2}{1000}<1, \\
& r(t, s)=\frac{30}{499} t s, \quad q(r)=\frac{6}{100} r, \quad t_{1}=s_{0}^{*}=.030061011, \\
& \eta=\frac{15}{499}, \quad \beta=\frac{514}{499} .
\end{aligned}
$$

Relations (11) and (12) become respectively

$$
\begin{aligned}
& r^{7}+.030061011 r^{6}+229.2611598 r^{5}+2.918374609 r^{4}-3141.619606 r^{3} \\
& +94.44026784 r^{2}-33888.20112 r+1018.713654 \leq 0
\end{aligned}
$$

and

$$
R \leq 5.688635222=R_{0} .
$$

That is, the hypotheses of Theorem 1 will be satisfied if we choose

$$
R_{1}=.030061013 \text { and } R=R_{0} .
$$


The conclusions of Theorem 1 can now follow.

\section{References}

[1] M. Altman, Iterative methods of higher order, Bull. Acad. Polon. Sci. Ser. Math. Astr. Phys. 9(1961), 63-68.

[2] I. K. Argyros, Quadratic equations and applications to Chandrasekhar's and related equations, Bull. Austral. Math. Soc. 32(1985), 275-292.

[3] - On a class of nonlinear integral equations arising in neutron transport, Aequationes Mathematicae 36(1988), 94-111.

[4] - On the solution of equations with nondifferentiable operators and the Ptak error estimates, BIT 90(1990), 752-754.

[5] —- On the convergence of a Halley-Chebysheff-type method under Newton-Kantorovich hypotheses, Appl. Math. Letters 6(1993), 71-74.

[6] I. K. Argyros and D. Chen, A note on the Halley method in Banach spaces, Appl. Math. and Comp. 58(1993), 215-224.

[7] -- On the midpoint iterative mehtod for solving nonlinear operator equations and applications to the solution of integral equations, Mathematica-Revue D'analyse Numerique et de Theorid de l'approximation, Tome 23, fasc. 2(1994), 139-152.

[8] I. K. Argyros and F. Szidarovszky, On the monotone convergence of general Newton-like methods, Bull. Austral. Math. Soc. 45(1992), 489-502.

[9] — The Theory and Application of Iteration Methods, C. R. C. Press, Inc. Boca Raton, Florida, (1993).

[10] S. Chandrasekhar, Radiative Transfer, Dover Publ., New York, 1960.

[11] X. Chen and T. Yamamoto, Convergence domains of certain iterative methods for solving nonlinear equation, Number. Funct. Anal. and Optimiz. 10(1989), 37-48.

[12] S. Kanno, Convergence theorems for the method of tangent hyperbolas, Math Japonica 37(1992), 711-722.

[13] L. V. Kantorovich, G. P. Akilov, Functional Analysis in Normed Spaces, Pergamon Press, New York, 1964.

[14] M. A. Mertvecova, An analog of the process of tangent hyperbolas for general functional equations (Russian), Dokl. Akad. Nauk. SSSR. 88(1953), 611-614.

[15] M. T. Necepurenko, On Chebysheff's method for functional equations (Russian), Usephi Mat. Nauk. 9(1954), 163-170.

[16] F. A. Potra and V. Ptak, Sharp error bounds for Newton's process, Numer. Math: 34(1980), 63-72.

[17] F. A. Potra, On an iterative algorithm of order $1.839 \cdots$ for solving nonlinear operator equations, Numer. Funct. Anal. and Optimiz. 7(1984-85), 75-106.

[18] R. A. Safiev, The method of tangent hyperbolas, Sov. Math. Dokl. 4(1963), 482-485.

[19] A. E. Taylor, Introduction to Functional Analysis, Wiley Publ. New York, 1957.

[20] S. Ul'm, Iteration methods with divided differences of the second order (Russian), Dokl. Akad. Nauk. SSSR 158(1964), 55-58. Soviet Math. Dokl. 5, 1187-1190.

[21] T. Yanamoto, On the method of tangent hyperbolas in Banach spaces, J. Comput. Appl. Math. 21(1988), 75-86.

[22] P. P. Zabrejko and D. F. Nguen, The majorant method in the theory of Newton-Kantorovich approximations and the Ptak error estimates, Numer. Funct. Anal. and Optimiz. 9(1987), 671-684. 